\title{
Evidências de validade do Zulliger-SC para avaliação do relacionamento interpessoal de crianças
}

\author{
Fabiola Cristina Biasi \\ Anna Elisa de Villemor-Amaral \\ Universidade São Francisco \\ São Paulo, SP, Brasil
}

\begin{abstract}
Resumo
Objetivou-se examinar evidências de validade de critério para o teste de Zulliger-Sistema Compreensivo (ZSC), tendo como foco os indicadores de relacionamento interpessoal. 119 crianças, de ambos os sexos, do quarto ao sexto ano do ensino fundamental, responderam a um sociograma para a seleção de dois grupos de participantes: o primeiro contendo 26 , classificadas como mais populares e 22 classificadas como menos populares. Esses dois grupos responderam ao ZSC. Considerou-se para a análise as seguintes variáveis: M, FM, m; a e p; COP e AG; GHR e PHR; FT, TF e T; Food; PER; PureH, SumH e Índice de Isolamento. Os indicadores, mais frequentes nas crianças mais populares com diferenças estatisticamente significativas, foram $\mathrm{Mp}(p=0,04)$, $\mathrm{p}(p=0,05)$ e marginalmente significativa PureH $(p=0,06)$. No grupo de crianças menos populares a maior frequência estatisticamente significativa foi $\mathrm{H}<(\mathrm{H})+\mathrm{Hd}+(\mathrm{Hd})\left(x^{2}=4,16, p=0,04\right)$. Conclui-se que quatro variáveis contribuíram para discriminar os grupos, $\mathrm{o}$ que evidencia validade de critério.
\end{abstract}

Palavras-chave: Avaliação Psicológica; ZSC; Crianças; Evidência de Validade.

\section{Evidence of validity of Zulliger-SC to the assessment of children's interpersonal relationship}

\begin{abstract}
This study aimed to examine criterion validity evidence for ZSC, focusing on interpersonal relationships indicators. 119 primary school children of both sexes from the fourth to the sixth year responded to the sociogram which allowed to select two groups of participants: the first containing 26 classified as the most popular and 22 classified as less popular. These two groups responded to the ZSC. The analysis considered: M, FM, m; a, p; COP, AG; GHR, PHR; FT, TF, T; Food; PER; PureH, SumH and Index Isolation rates. The indicators that showed statistically significant differences, higher among the most popular children, were $\mathrm{Mp}(p=0.04), \mathrm{p}(p=0.05)$ and PureH $(p=0.06)$ marginally significant. In the less popular group the highest statistically significant was $\mathrm{H}<(\mathrm{H})+\mathrm{Hd}+(\mathrm{Hd})\left(x^{2}=4.16, \mathrm{p}=0.04\right)$. In conclusion, four variables contributed to discriminate groups, which contributes to demonstrate criterion validity.
\end{abstract}

Keywords: Psychological Assessment; ZSC; Children; Validity Evidence.

\section{Evidencias de validez de la prueba de Zulliger-sc para la evaluación de relación interpersonal de niños}

\section{Resumen}

El objetivo del trabajo fue examinar evidencias de validez de criterio para el test ZSC, cuyo foco fueron los indicadores de relacionamiento interpersonal. 119 niños de ambos sexos, del cuarto al sexto año de educación primaria, respondieron al sociograma lo que permitió la selección de dos grupos de participantes: el primer contiene 26 clasificados como los más populares y el otro 22 clasificados como menos populares. Estos dos grupos respondieron a el ZSC. Se consideraron para el análisis los indicadores: M, FM, m; a e p; COP e AG; GHR e PHR; FT, TF e T; Food; PER: PureH, SumH e Índice de Aislamiento. Aquellos que revelaron diferencia estadística significativa, con mayor frecuencia entre los niños más populares fueron: $\mathrm{Mp}(p=0,04), \mathrm{p}(p=0,05)$ y $\mathrm{PureH}$ $(p=0,06)$ fue marginalmente significativa. En el grupo de niños menos populares la mayor frecuencia fue: $\mathrm{H}<(\mathrm{H})+\mathrm{Hd}+(\mathrm{Hd})$ $\left(x^{2}=4,16, \mathrm{p}=0,04\right)$. Se concluye que cuatro variables contribuyeron a discriminar grupos, lo que demuestra su contribución para la evidencia de validez de criterio.

Palabras clave: Evaluación Psicológica, ZSC; Niños; Evidencia de Validez. 


\section{Introdução}

O processo de avaliação quando realizado precocemente, favorece o desenvolvimento equilibrado da criança, podendo ter caráter preventivo ao contribuir para a redução do aparecimento de psicopatologias (Perez-Ramos, 2000); identificar suas potencialidades e fragilidades e sugerir os encaminhamentos adequados (Schelini, Gomes \& Wechsler, 2006). A avaliação fornecerá dados que, integrados pelo profissional com seus conhecimentos sobre desenvolvimento infantil, personalidade e sofrimento mental, permitirão intervenções mais precoces e mais acertadas (Hisatugo \& Custódio, 2013).

Destaque deve ser dado às recomendações de Nunes, Teixeira e Deakin (2010) que enfatizam que a criança ainda está em processo de desenvolvimento e os resultados não podem ser considerados como taxativos, bem como as afirmações de Davoglio, Gauer, Jaeger e Tolotti (2012) de que não se pode descartar que cada fase evolutiva tem aspectos muito peculiares diretamente relacionados a manifestação e a formação da personalidade.

A avaliação precoce do relacionamento interpessoal pode proporcionar uma compreensão de como essas relações vem se estruturando no decorrer do desenvolvimento da criança e esse entendimento pode auxiliar na identificação de comportamentos psicopatológicos. Crianças que são expostas a experiências traumáticas - violência física ou psicológica, maus tratos, negligência, punição agressiva em excesso - estão mais suscetíveis a apresentarem traços ou sintomas de transtorno de personalidade. O transtorno pode aparecer em no mínimo duas das quatro áreas de sua vida, afetando a cognição, a afetividade, as relações interpessoais, ou o controle dos impulsos. Em se tratando do diagnóstico dos transtornos de personalidade, este só é feito ao final da adolescência de acordo com os critérios nosográficos, mas a avaliação e a identificação das áreas afetadas precocemente pode revelar características que possam progredir para quadros psicopatológicos (Davoglio, Gauer, Jaeger \& Tolotti, 2012).

No processo de avaliação psicológica é necessário que o psicólogo selecione os melhores instrumentos para compreender as características dos sujeitos, sendo de fundamental importância respeitar as características individuais, os objetivos da avaliação e o contexto da avaliação, considerando as demandas culturais, econômicas, sociais, educacionais e políticas (Hisatugo \& Custódio, 2013; Tavares, 2003). Schelini, Gomes e Wechsler (2006) acrescentam que a avaliação psicológica abrange várias técnicas como: entrevistas, observações, dinâmicas, aplicação de testes, entre outros, bem como a interpretação dos resultados obtidos e entrevista devolutiva.

Entre os testes, destacam-se as técnicas expressivas de personalidade, também chamadas de projetivas, Anzieu (1989) destaca que esses testes facilitam a investigação de processos mentais profundos, pois seus estímulos são pouco estruturados e favorecem a criação e elaboração de respostas com base mais em determinantes internos do que externos. Nunes, Teixeira e Deakin (2010) enfatizam que as técnicas expressivas tem por finalidade conhecer e compreender os afetos e os comportamentos, avaliar a personalidade, tanto os aspectos dinâmicos quanto estruturais do indivíduo e também as mudanças que podem ocorrer ao longo ou ao final do processo de avaliação/psicoterapia.

Esses testes continuam sendo alvo de muitas críticas, embora apresentem inúmeras vantagens no contexto da avaliação psicológica. As críticas recebidas são devido frequentemente a sua relação com o conceito de projeção da teoria psicanalítica, o que acaba por injustamente limitar o uso desses testes ao campo psicanalítico. Entretanto, tais instrumentos podem ser aplicáveis sob a perspectiva de outros escopos teóricos, possibilitando em várias circunstâncias um exame mais abrangente e profundo, avaliando traços e estados da personalidade como um todo (Bandeira, Trentini, Winck, \& Lieberknecht, 2006; Weiner, 2000).

Cabe destacar, que atualmente somente se têm quatro técnicas expressivas de personalidade aprovadas pelo Sistema de Avaliação de Testes Psicológicos (SATEPSI) para a realização de avaliação de crianças e adolescentes, são elas: o CAT (Teste de Apercepção Infantil - figuras de animais), Rorschach, o HTP (Casa, Árvore e Pessoa) e o Teste das Pirâmides Coloridas de Pfister, sendo que o ZSC ainda está em processo de normatização para a população de crianças e adolescentes e apresenta poucas pesquisas com está população, como se verá a seguir.

$\mathrm{O}$ teste de Zulliger foi criado por Hans Zulliger para selecionar oficiais suíços para a Segunda Guerra Mundial, tendo como base o Método de Rorschach, e se assemelha a este no que se refere às características de estímulos, aplicação, classificação e interpretação de respostas (Vaz, 1998; Villemor-Amaral \& Primi, 2009; Zulliger \& Salomon, 1970). Por sua vez, o Sistema Compreensivo foi desenvolvido por John Exner para o Método Rorschach ao reunir as contribuições dos cinco principais sistemas do Rorschach utilizados nos Estados Unidos, conservando aqueles indicadores cujos estudos traziam mais evidências de sua validade, 
e com isso aprimorando o método (Villemor-Amaral \& Primi, 2009). O teste de ZSC se propõe a investigar aspectos cognitivos e afetivos da personalidade (Zulliger \& Salomon, 1970), porém de um modo mais rápido e menos oneroso do que o Rorschach.

O Sistema Compreensivo abrange nove categorias amplas de codificação das respostas - localização; qualidade de desenvolvimento (DQ); qualidade formal (FQ); determinantes; conteúdos; populares; respostas pares; atividade organizativa; e códigos especiais. Essas variáveis de base são reorganizadas no sumário estrutural em mais sete agrupamentos relativos ao funcionamento psicológico: ideação, processamento, mediação, autoimagem, recursos e controle, afetividade e a categoria de relacionamento interpessoal. No contexto da avaliação clínica todos esses agrupamentos devem ser avaliados num conjunto dinâmico para que se tenha uma visão mais fiel do que se passa com o indivíduo, entretanto, numa situação de pesquisa como essa, vale destacar, alguns indicadores quando o interesse é buscar evidências de validade para as interpretações feitas com base nos mesmos. Como o foco dessa investigação destacouse o agrupamento de relacionamento interpessoal, que a seguir será melhor detalhado (Villemor-Amaral \& Primi, 2009).

Weiner (2000) define o relacionamento interpessoal como o modo com que as pessoas estabelecem os vínculos com os outros, sendo determinado pelas atitudes e grau de interações. O autor caracteriza os relacionamentos interpessoais pelas capacidades de:

1) manter um nível de interesse, envolvimento e conforto razoável na interação com as pessoas; 2) antecipar intimidade e segurança nas interações interpessoais; 3) equilibrar a colaboração e a aquiescência, por um lado, e a competitividade e a assertividade, por outro, no relacionamento com os demais; 4) perceber o outro e as situações sociais de modo preciso e empático (p. 156).

Os indicadores de relacionamento interpessoal do ZSC tal como no Rorschach - Sistema Compreensivo, são os determinantes de movimento, fortemente impregnados de conteúdo projetivo uma vez que trata-se de movimento atribuído a imagens estáticas (Villemor-Amaral \& Primi, 2009). Para Weiner (2000), o movimento humano (M) revela a capacidade de empatia, que pode ser entendida como a habilidade que o indivíduo tem de se colocar no lugar dos outros. Indica ainda a percepção social do indivíduo, bem como a sua capacidade de formar impressões realistas das pessoas e das relações interpessoais. De acordo com
Villemor-Amaral e Primi (2009), movimento animal (FM) e inanimado (m) revelam um distanciamento da identificação com o humano.

Outro indicador que faz parte do agrupamento relacionamento interpessoal é o Movimento Cooperativo (COP) que, quando superior a Movimento Agressivo (AG) leva ao pressuposto que o indivíduo tenha uma disposição a conceber os vínculos de maneira mais positiva. Quando AG supera COP isso sugere uma concepção mais hostil sobre como as pessoas se relacionam, o que pode ou não se refletir em seu comportamento social. Todos os determinantes de movimento também serão classificados como ativo (a) e passivo (p), sendo que os indivíduos que tendem a assumir as responsabilidades e serem autônomos em suas decisões tem aumento de movimento ativo, enquanto aqueles que são mais omissos tem a predominância de movimentos passivos (Exner \& Sendin, 1999; Villemor-Amaral \& Primi, 2009).

Boas representações humanas (GHR) são, de acordo com Viglione, Perry e Meyer (2003), também indicadores de contato interpessoal mais adaptado, sendo esperados em adultos com maior frequência do que as representações humanas pobres (PHR). Isso representa a capacidade de ver a si mesmo de modo real e integrado. Caso contrário, supõe-se percepções distorcidas e negativas das relações com os demais.

Conforme descrito por Exner e Sendin (1999), as respostas de textura $\mathrm{FT}$, TF e $\mathrm{T}$ dizem respeito às necessidades de contato emocional e expressam os sentimentos de solidão, abandono ou privação afetiva. Se o somatório de todas as respostas de textura (SumT) estiver aumentado, é preciso averiguar se nos últimos meses a pessoa viveu perdas afetivas que podem influenciar o aumento.

$\mathrm{O}$ indicador $\mathrm{Fd}(\mathrm{Food})$ revela tendência em emitir comportamentos dependentes. De acordo com o que argumentam Exner e Sendin (1999), essas pessoas esperam que os outros resolvam seus problemas e satisfaçam suas necessidades, especialmente se esse indicador venha também associado às variáveis passivo e textura.

Exner e Sendin (1999) enfatizam que nos protocolos de crianças e adolescentes o Índice de Isolamento, outro dado relevante nessa pesquisa, merece maior destaque, visto que nesse período o relacionamento com os outros é um fator essencial para o desenvolvimento equilibrado do indivíduo. Esse índice envolve as variáveis botânica (Bt), nuvem $(\mathrm{Cl})$, geografia $(\mathrm{Ge})$, paisagem $(\mathrm{Ls})$ e natureza $(\mathrm{Na})$, é um indicador fundamental para verificar a percepção do meio interpessoal, podendo revelar isolamento ou retraimento social. 
$\mathrm{O}$ indicador Respostas Personalizadas (PER) também compõe o agrupamento de relacionamento interpessoal. Conforme a criança amadurece sua frequência tende a diminuir e até desaparecer. Em adultos, seu aumento indica infantilidade ou imaturidade (Exner \& Sendin, 1999).

As repostas de conteúdo humano inteiro e real, $\mathrm{H}$, sugerem interesse pelas pessoas, no entanto, quando sua frequência é baixa podem revelar dificuldades na identificação com os outros. O SumH é outro indicador que compreende a soma de todos conteúdos humanos inteiros $-\mathrm{H}$, conteúdos para-humanos $-(\mathrm{H})$, detalhes humano - Hd e detalhes para-humanos - $(\mathrm{Hd})$. Quando $\mathrm{H}<(\mathrm{H})+\mathrm{Hd}+(\mathrm{Hd})$ pressupõe-se que o indivíduo se identifique com figuras parciais ou fictícias, havendo uma concepção imatura de si mesmo e do objeto (Villemor-Amaral \& Primi, 2009).

Ainda não existem na literatura nacional e interncional estudos que demonstrem evidências de validade de critério para esses indicadores no ZSC, principalmente considerando a população infantil. Conforme a American Educational Rersearch Association; American Psychological Association; National Council On Measurement In Education (1999) e Urbina (2007) a validade é um fator essencial para garantir rigor psicométrico aos testes, de tal modo que cada novo indicador precisa ser estudado quanto às suas qualidades psicométricas. Os procedimentos para esse fim podem também ser aplicados às técnicas expressivas de personalidade. No presente trabalho priorizou-se a validade de critério descrita por Primi, Muniz e Nunes (2009) como aquela que demonstra relação entre a eficácia com que o teste prevê variáveis externas ou variáveis de critério. A associação entre teste e o critério antecipa a aplicação de uma variável, e a relação estabelecida com o conteúdo avaliado, tendo por finalidade prognosticar eventos objetivos para determinados contextos.

Optou-se por utilizar como critério para mensurar as preferências e rejeições das crianças a aplicação do sociograma. Tal escolha foi pautada nos postulados de Moreno (1953/2008) que aponta esse instrumento como um bom indicador das preferências e rejeições no meio social. A análise de seus resultados revela a estrutura social, ou seja, é possível observar as relações estabelecidas entre os indivíduos, sendo que tais relações envolvem aspectos afetivos, responsáveis por estruturar as preferências e rejeições dentro dos grupos, o que justifica a escolha desse procedimento na condução desta pesquisa.

Na revisão de literatura realizada nas bases de dados SciELO, PePSIC e PsycINFO foram encontrados três estudos tendo o Zulliger como instrumento aplicado em crianças e adolescentes, dois internacionais e um nacional, descritos a seguir. Uhinki, Mattlar, Sandahl, Vesala e Carlsson (1990) aplicaram o Zulliger em 50 adolescentes finlandeses de 15 a 17 anos. Os resultados demonstraram uma menor quantidade de respostas de movimento humano $\mathrm{M}$ no grupo de adolescentes $(\mathrm{X}=1,3)$ quando comparadas ao grupo de adultos não pacientes $(X=1,5)$. As somas de movimento ativo $(X=1,1)$ foi menor que passivo $(X=1,3)$ no grupo de adolescentes, revelando tendência cognitiva passiva e dependente. A conclusão a que os autores chegaram a partir dos resultados é que adolescentes diferem dos adultos, entretanto, não foram apresentadas análises estatísticas de significância. Os resultados do Zulliger apontaram que a estrutura de personalidade dos adolescentes equivale ao que é esperado nessa fase de desenvolvimento, demonstrando que os adolescentes têm capacidades empáticas, com certa imaturidade, que foi identificado por um dos indicadores de relacionamento interpessoal, o movimento humano.

Lis, Magro e Rossi (1990) compararam os resultados do Zulliger e do Rorschach de 120 crianças de ambos os sexos de 5 anos e 6 meses a 10 anos e 5 meses. Cabe mencionar que os autores não esclareceram qual foi o Sistema utilizado para interpretar as respostas. Os dados do Rorschach e do Zulliger foram analisados separadamente, considerando o sexo e idade. No Rorschach os indicadores que aumentaram significativamente com a idade foram M (movimento humano) e FM (movimento animal). Já os indicadores A (animal), Pl (plantas) e Arch (arquitetura) apresentaram as médias diminuídas com a idade. Com relação ao sexo os indicadores que tiveram resultados estatisticamente significativos no Rorschach foram $\mathrm{W}+\%$ (Respostas de localização $\mathrm{W}$ associadas à qualidade de desenvolvimento sintetizadas) e M, sendo estes mais frequentes no sexo feminino, enquanto que para o sexo masculino, o indicador mais frequente foi o $\mathrm{H}$ (frequência de respostas de conteúdo humano inteiro). No Zulliger foi também o $\mathrm{M}$ a variável que teve valores significativamente aumentados de acordo com a idade, enquanto que o A tendia a diminuir com a idade. A variável sexo não mostrou diferenças estatisticamente significativas no Zulliger. Os resultados revelam que com o aumento da idade, aumenta também o interesse pelas pessoas, demonstrando que ambos os testes são capazes de revelar o amadurecimento progressivo por meio de seus indicadores.

Tavella e Villemor-Amaral (2014) aplicaram o ZSC, e o correlacionaram com a criatividade identificada pelo Teste de Criatividade Figura Infantil (TCFI), em 90 crianças de idades entre 11 e 12 anos, 
de ambos os sexos e de escolas públicas. O intuito não era destacar aspectos de relacionamento interpessoal, porém, ao comparar crianças mais e menos criativas, as mais criativas revelaram mais respostas de movimento humano ativo (Ma). Dentro do contexto da criatividade, esse indicador estaria relacionado com maior contato com a vida interior, porém o M também revela um senso de identidade mais bem desenvolvido, o que repercute significativamente na qualidade dos relacionamentos. Indiretamente, pode-se constatar nessa pesquisa que as crianças mais criativas têm, concomitantemente, capacidades empáticas e podem ser mais autônomas em suas decisões.

Além desses três estudos realizados com a população de crianças e adolescentes é importante mencionar pesquisas que abordaram a questão do relacionamento interpessoal no teste de ZSC na população adulta, uma pesquisa internacional e uma nacional. Sandahl, Mattlar, Carlsson, Vesala e Rosenqvist (1990) aplicaram o Zulliger e compararam a estrutura de personalidade de adultos europeus. Um grupo foi formado por 22 adultos não pacientes, o outro grupo por 36 adultos que participavam de um projeto social. Esses dois grupos foram comparados ao grupo formado por 700 não pacientes de Exner. $\mathrm{O}$ grupo de não pacientes obteve uma proporção de respostas a:p igual a 17:9, indicando flexibilidade cognitiva e maior autonomia e independência.

O estudo nacional de Grazziotin e Scortegagna (2012) verificou evidências de validade no ZSC, tendo como objetivo correlacionar os indicadores de relacionamento interpessoal com Inventário de Habilidades Sociais (IHS). Foram sujeitos da pesquisa 19 pessoas, 10 eram do sexo feminino, com idades entre 18 e 43 anos, de um supermercado. Os resultados demonstraram correlações positivas entre GIHS (índice geral de habilidades sociais), F1 (autoafirmação e enfrentamento com risco) e F3 (conversação e desenvoltura social) com GHR (boas representações humanas) e SumH (soma de conteúdo humano). Encontraram correlações negativas entre as variáveis F5 (autocontrole da agressividade) e GIHS com AG (movimento agressivo).

Finalmente convém destacar dois estudos, que utilizaram os mesmos indicadores de relacionamento interpessoal explorados nesta pesquisa só que por meio do Método de Rorschach no Sistema Compreensivo, fonte que deu origem ao ZSC. O estudo realizado por Resende, Carvalho e Martins (2012) investigou o desempenho de crianças e adolescentes no Rorschach Sistema Compreensivo. Participaram da pesquisa 201 crianças e adolescentes com idades entre 5 a 14 anos. Os resultados dos adolescentes de 12 a 14 anos apontam aumento na frequência de Movimentos Humanos, Conteúdos Humanos e Respostas Boas de Representações Humanas, indicando habilidade em se perceber e perceber ao outro e capacidade em se relacionar com os demais. Os resultados de forma geral apontaram que com o aumento da idade os indivíduos tendem a dar respostas mais complexas e precisas no Rorschach, indicando maior capacidade cognitiva, autopercepção, mais envolvimento nos relacionamentos e capacidade em discriminar e modular as emoções.

O estudo de Balbi e Queiroz (2012) teve como foco a validade do índice de déficit relacional (CDI), que avalia o relacionamento interpessoal no Rorschach. Esse índice contém os principais indicadores de relacionamento interpessoal também destacados no ZSC, embora este não contenha CDI no seu esquema interpretativo. Esses pesquisadores revelaram que no Rorschach pessoas com CDI 4 ou 5 apresentam dificuldades de relacionamento. Participaram da pesquisa 8 universitários que obtiveram resultados positivos para o CDI, que responderam ao Rorschach e a uma entrevista qualitativa. A entrevista continha perguntas sobre dados sociodemográficos, histórico pessoal, demandas sociais e relacionamento passados e atuais com pais, irmãos e irmãs. Os dados qualitativos da entrevista confirmaram o resultado positivo no CDI, indicando dificuldades em se relacionar com os demais, distanciamento com relação ao pai, falta de intimidade com irmãos, e desentendimento com a mãe.

Com base no exposto até o momento esta pesquisa partiu da hipótese de que os indicadores de relacionamento interpessoal no ZSC poderiam diferenciar crianças mais populares das menos populares entre os colegas. Selecionou-se, assim, as variáveis relacionadas com a capacidade de empatia, que informam sobre impressões mais realistas dos outros e de si mesmo, necessidade de contato emocional, autonomia e responsabilidade.

O objetivo desse estudo é ampliar as evidências de validade para o ZSC, com foco na avaliação do relacionamento interpessoal de crianças, averiguando diferenças na frequência das referidas variáveis em crianças mais populares e menos populares, conforme resultado do sociograma.

\section{Método}

\section{Participantes}

Fizeram parte da pesquisa 119 crianças que participaram da primeira etapa, respondendo a um sociograma. As crianças eram de duas escolas públicas 
de uma cidade do interior do estado de São Paulo, do quarto, quinto e sexto ano do ensino fundamental, com idades entre 9 a 14 anos, sendo 66 do sexo feminino. Para a segunda etapa, foram selecionadas 48 crianças com base nos resultados extremos e opostos obtidos no sociograma, que responderam ao ZSC. Desse modo, a amostra foi dividida em dois grupos.

O grupo 1 foi formado por 26 crianças que obtiveram maior quantidade de preferências no sociograma, destas 18 eram do sexo feminino. Este grupo foi denominado grupo de crianças populares. $\mathrm{O}$ grupo 2 foi formado por 22 crianças que obtiveram maior quantidade de rejeições no sociograma, destas 14 eram do sexo masculino. Este grupo foi denominado grupo de crianças menos populares.

\section{Instrumentos}

Sociograma - O teste sociométrico ou sociograma tem por finalidade revelar o grau de organização dos grupos (Moreno, 1953/2008). A técnica sociométrica clássica permite ao indivíduo escolher as pessoas com quem gostaria de realizar uma atividade, as escolhas devem ser ordenadas respeitando as preferências, ou seja, o mais escolhido recebe a pontuação mais alta, esta pontuação vai diminuindo até chegar a última (Moreno, 1953/2008).

Neste caso as perguntas do estudo foram elaboradas pela pesquisadora com a finalidade de determinar o grau de preferência e rejeição entre os colegas, sendo que para as crianças do quarto e quinto ano do ensino fundamental eram: 1) Quais colegas da sala você gostaria de convidar para brincar? 2) Quais colegas da sala você não gostaria de convidar para brincar? Já para as crianças do sexto ano, as perguntas foram as seguintes: 1) Quais colegas da sala você gostaria de convidar para bater papo? 2) Quais colegas da sala você não gostaria de convidar para bater papo? Cabe destacar que as perguntas para as crianças do sexto ano foram modificadas, pois as brincadeiras mudam nessa faixa etária, por isso optou-se por excluir o brincar e incluir o bater papo, já que podem preferir estar com os colegas para essa finalidade.

As perguntas número 1, foram pontuadas positivamente, ou seja, +3 para o primeiro colega, +2 para o segundo e +1 para o terceiro escolhido. As perguntas número 2 , receberam pontuações negativas, -3 para o colega mais rejeitado, -2 para o segundo mais rejeitado e -1 para o terceiro. As crianças foram informadas de que as suas escolhas deveriam respeitar o mais preferido e o aquele que elas não gostariam de convidar para as atividades. O tempo de aplicação foi de aproximadamente 15 minutos. No que se refere à quantidade de escolhas, optou-se por reduzir para três o número de escolhas, facilitando o tratamento estatístico. Essa estrutura já foi utilizada em dois estudos realizados por Bartholomeu, Carvalho, Silva, Miguel e Machado (2011) e Bartholomeu, Montiel e Pessotto (2011).

As crianças que participaram da segunda etapa da pesquisa, a aplicação do ZSC, deveriam ter obtido pontuação mínima de +6 e -6 pontos. Elas poderiam ter recebido duas pontuações de mais preferido ou mais rejeitado, ou então ter recebido várias escolhas de preferência ou rejeição em qualquer ordem, desde que obtivessem +6 ou -6 pontos, a pontuação máxima foi de $+14,-31$. A partir desses resultados formou-se dois grupos, grupo 1 de crianças populares e grupo 2 de crianças menos populares.

Zulliger-Sistema Compreensivo - O Teste ZSC avalia aspectos da personalidade e é composto por três pranchas com manchas de tinta simétricas e uma folha de resposta. Utilizou-se como base para correção e interpretação o Sistema Compreensivo. A aplicação é realizada em dois momentos: o primeiro é denominado como associação livre em que as três pranchas são apresentadas e o sujeito deve responder a questão "com que isso se parece?" ou "o que poderia ser?". As respostas são anotadas pelo examinador. No segundo momento, denominado inquérito, apresenta-se novamente os cartões, as respostas dadas são repetidas e o sujeito deve responder "onde viu e o que deu aquela ideia", neste momento o examinador anota a localização na folha de resposta e também o que fez associar a tal conceito. O tempo de aplicação é de aproximadamente 30 minutos (Villermor-Amaral \& Primi, 2009).

O Sistema Compreensivo foi utilizado para a aplicação, codificação e interpretação das respostas. Um dos critérios desse Sistema é o preenchimento do sumário estrutural, que envolve frequências, porcentagens ou proporções, estas por sua vez são agrupadas em sete categorias, sendo três relativas ao funcionamento cognitivo: processamento, mediação e ideação e quatro relacionados à dinâmica afetiva, autoimagem, capacidade de relacionamento interpessoal e ao controle e tolerância ao estresse. Para a presente pesquisa, utilizou-se apenas os indicadores do agrupamento do relacionamento interpessoal.

\section{Procedimentos}

Inicialmente obteve-se autorização da secretaria municipal de ensino, posteriormente o projeto foi encaminhado ao Comitê de Ética da Universidade 
São Francisco, após a aprovação os Termos de Consentimento Livre e Esclarecido (TCLE) foram enviados aos pais, juntamente com uma carta explicativa. As crianças cujos pais consentiram e que quiseram participar responderam ao sociograma.

As aplicações do sociograma foram geralmente coletivas e individuais para aquelas crianças que não estavam presentes nas aplicações coletivas. As crianças receberam duas folhas, a pesquisadora solicitou que fossem preenchidos os dados de identificação e na sequência foram lidas as seguintes perguntas "1) Quais colegas da sala você gostaria de convidar para brincar? ou 1) Quais colegas da sala você gostaria de convidar para bater papo?" cada criança escolheu três colegas, obedecendo uma ordem de preferência. A segunda folha continha mais uma pergunta a qual responderam: “2) Quais colegas da sala você não gostaria de convidar para brincar? ou 2) Quais colegas da sala você não gostaria de convidar para bater papo?", as quais as crianças tinham que responder escolhendo três colegas de sala obedecendo uma ordem de rejeição para cada pergunta. A pesquisadora mencionou que as respostas não seriam divulgadas a menos que cada um quisesse contar as suas escolhas. O tempo de aplicação foi de aproximadamente 15 minutos.

Posteriormente as respostas foram analisadas e de acordo com os resultados dois grupos foram formados: de crianças populares que foram mais pontuadas pelos colegas em todas as perguntas (aquelas que são convidadas para brincar ou bater papo) e o de crianças menos populares (por nunca serem convidadas para brincar ou bater papo). Esses dois grupos de crianças responderam ao ZSC.

As aplicações do ZSC foram individuais e o tempo de aplicação foi de aproximadamente de 30 minutos. As aplicações ocorreram em sala disponibilizada pela escola.

Em seguida os protocolos foram codificados pela pesquisadora às cegas e revistos pela orientadora. Posteriormente, selecionou-se $25 \%$ dos protocolos para serem analisados por um juiz independente, com a finalidade de verificar a fidedignidade dos dados desta pesquisa. Na sequência, realizou-se estatística descritiva, dos indicadores de relacionamento interpessoal por grupos; o Teste $U$ de Mann-Whitney para testar diferenças estatisticamente significativas entre grupos e o Teste de Qui-Quadrado para os dados categóricos, o programa SPSS (Statiscal Package for Social Sciense) foi utilizado para realizar as análises estatísticas descritas acima.

\section{Resultados}

O Coeficiente Kappa foi utilizado para analisar a precisão dos dados desta pesquisa, de modo que foram realizadas duas codificações às cegas do ZSC pela pesquisadora e por um juiz independente com bom conhecimento neste teste. Os valores de concordância estavam na maioria entre 0,85 e 1,00 . Os critérios de confiabilidade menores de 0,20 são considerados pobres, entre 0,21 a 0,40 suficientes, entre 0,41 a 0,60 como moderados, entre 0,61 e 0,80 bons e entre 0,81 a 1,00 excelentes (Landis \& Koch, 1977). Verificase que os dados desta pesquisa são fidedignos, pois apresentaram resultados bons e excelentes pelo coeficiente Kappa. Na Tabela 1 são apresentadas as estatísticas descritivas e as comparações de médias das variáveis do relacionamento interpessoal por meio do Teste $U$ de Mann-Whitney.

Na Tabela 1, estão expressos os desempenhos dos grupos de crianças populares (grupo 1) e de crianças menos populares (grupo 2) nas variáveis do relacionamento interpessoal, por meio da média, desvio padrão, e valor mínimo e máximo. A tabela expõe também os resultados do teste $U$ de Mann-Whitney que foi utilizado para testar se existem diferenças estatisticamente significativas entre as médias dos grupos de crianças populares e menos populares. Os resultados apontaram 3 diferenças estatisticamente significativas das 21 possíveis, que foram nos indicadores $\mathrm{p}$ e $\mathrm{Mp}$, e marginalmente significativo na variável PureH.

Os resultados demonstram que há uma tendência das crianças mais populares de darem mais respostas de movimento humano que as menos populares, embora somente a frequência de movimentos passivos e movimentos humanos passivos tenha sido significativamente maior nesse grupo. O grupo de crianças populares revelou média significativamente maior na variável movimentos passivos, $\mathrm{p},(p=0,05)$, e também no determinante de Movimento Humano passivo, Mp, $(p=0,04)$ quando comparado ao grupo de crianças menos populares. $\mathrm{O}$ grupo de crianças populares revelou ainda média maior e marginalmente significativa no indicador conteúdo humano inteiro, PureH, $(p=0,06)$.

$\mathrm{Na}$ sequência realizou-se o Teste Qui-quadrado para as variáveis categóricas, ou seja, na forma de frequência, comparando-se os desempenhos dos grupos. $\mathrm{O}$ grupo de crianças menos populares demonstrou resultado estatisticamente significativo no indicador $\mathrm{H}<(\mathrm{H})+\mathrm{Hd}+(\mathrm{Hd})\left(x^{2}=4,16, p=0,04\right)$. Este grupo obteve frequência maior de conteúdos para-humanos, detalhes humanos e detalhes para-humanos. 
TABELA 1

Estatística descritiva e análise comparativa das médias dos indicadores do relacionamento interpessoal

\begin{tabular}{|c|c|c|c|c|c|c|c|c|c|c|}
\hline Variáveis & Grupos & $N$ & Min & $\operatorname{Max}$ & Media & $D P$ & Mediana & Moda & $\begin{array}{c}\text { Teste de } \\
\text { Independência } \\
\text { (Ude Mann-Whitney) }\end{array}$ & Sig. \\
\hline \multirow{2}{*}{ M } & Grupo 1 & 26 & 0,00 & 5,00 & 1,00 & 1,20 & 1,00 & 0,00 & \multirow{2}{*}{$\begin{array}{c}U=225,50 \\
\mathrm{z}=-1,365\end{array}$} & \multirow{2}{*}{0,17} \\
\hline & Grupo 2 & 22 & 0,00 & 1,00 & 0,50 & 0,51 & 0,50 & 0,00 & & \\
\hline \multirow{2}{*}{ Мa } & Grupo 1 & 26 & 0,00 & 3,00 & 0,69 & 0,79 & 1,00 & 0,00 & \multirow{2}{*}{$\begin{array}{c}U=247,00 \\
z=-0,907\end{array}$} & \multirow{2}{*}{0,36} \\
\hline & Grupo 2 & 22 & 0,00 & 1,00 & 0,45 & 0,51 & 0,00 & 0,00 & & \\
\hline \multirow{2}{*}{$\mathrm{Mp}$} & Grupo 1 & 26 & 0,00 & 2,00 & 0,35 & 0,63 & 0,00 & 0,00 & \multirow{2}{*}{$\begin{array}{c}U=221,00 \\
\mathrm{z}=-2,08\end{array}$} & \multirow{2}{*}{0,04} \\
\hline & Grupo 2 & 22 & 0,00 & 1,00 & 0,05 & 0,21 & 0,00 & 0,00 & & \\
\hline \multirow{2}{*}{ FM } & Grupo 1 & 26 & 0,00 & 3,00 & 0,54 & 0,90 & 0,00 & 0,00 & \multirow{2}{*}{$\begin{array}{c}U=255,50 \\
z=-0,724\end{array}$} & \multirow{2}{*}{0,47} \\
\hline & Grupo 2 & 22 & 0,00 & 4,00 & 0,73 & 1,08 & 0,00 & 0,00 & & \\
\hline \multirow{2}{*}{ FMa } & Grupo 1 & 26 & 0,00 & 3,00 & 0,42 & 0,76 & 0,00 & 0,00 & \multirow{2}{*}{$\begin{array}{c}U=258,00 \\
\mathrm{z}=-0,69\end{array}$} & \multirow{2}{*}{0,49} \\
\hline & Grupo 2 & 22 & 0,00 & 3,00 & 0,55 & 0,80 & 0,00 & 0,00 & & \\
\hline \multirow{2}{*}{ FMp } & Grupo 1 & 26 & 0,00 & 1,00 & 0,12 & 0,33 & 0,00 & 0,00 & \multirow{2}{*}{$\begin{array}{c}U=280,50 \\
z=-0,22\end{array}$} & \multirow{2}{*}{0,83} \\
\hline & Grupo 2 & 22 & 0,00 & 3,00 & 0,19 & 0,66 & 0,00 & 0,00 & & \\
\hline \multirow{2}{*}{$\mathrm{m}$} & Grupo 1 & 26 & 0,00 & 2,00 & 0,15 & 0,53 & 0,00 & 0,00 & \multirow{2}{*}{$\begin{array}{c}U=270,00 \\
z=-0,625\end{array}$} & \multirow{2}{*}{0,53} \\
\hline & Grupo 2 & 22 & 0,00 & 2,00 & 0,23 & 0,61 & 0,00 & 0,00 & & \\
\hline \multirow{2}{*}{$\mathrm{ma}$} & Grupo 1 & 26 & 0,00 & 1,00 & 0,08 & 0,28 & 0,00 & 0,00 & $\mathrm{U}=282,00$ & 0,86 \\
\hline & Grupo 2 & 22 & 0,00 & 1,00 & 0,09 & 0,29 & 0,00 & 0,00 & $\mathrm{z}=-0,17$ & 0,80 \\
\hline $\mathrm{mp}$ & Grupo 1 & 26 & 0,00 & 1,00 & 0,07 & 0,28 & 0,00 & 0,00 & $\mathrm{U}=281,00$, & 0,82 \\
\hline mp & Grupo 2 & 22 & 0,00 & 2,00 & 0,14 & 0,47 & 0,00 & 0,00 & $\mathrm{z}=-0,22$ & 0,02 \\
\hline a & Grupo 1 & 26 & 0,00 & 4,00 & 1,20 & 1,13 & 1,00 & 1,00 & $\mathrm{U}=276,00$ & 0,83 \\
\hline a & Grupo 2 & 22 & 0,00 & 4,00 & 1,09 & 1,02 & 1,00 & 1,00 & $z=-0,22$ & 0,83 \\
\hline & Grupo 1 & 26 & 0,00 & 3,00 & 0,62 & 0,85 & 0,00 & 0,00 & $U=210,50$ & 0,05 \\
\hline $\mathrm{p}$ & Grupo 2 & 22 & 0,00 & 5,00 & 0,36 & 1,14 & 0,00 & 0,00 & $z=-1,953$ & 0,05 \\
\hline SumT & Grupo 1 & 26 & 0,00 & 1,00 & 0,04 & 0,20 & 0,00 & 0,00 & $\mathrm{U}=275,00$ & 036 \\
\hline & Grupo 2 & 22 & 0,00 & 0,00 & 0,00 & 0,00 & 0,00 & 0,00 & $\mathrm{z}=-0,92$ & 0,50 \\
\hline PureH & Grupo 1 & 26 & 0,00 & 4,00 & 1,35 & 0,85 & 1,35 & 1,00 & $\mathrm{U}=200,00$ & 0.06 \\
\hline Рuren & Grupo 2 & 22 & 0,00 & 2,00 & 0,86 & 0,77 & 1,00 & 1,00 & $\mathrm{z}=-1,91$ & 0,00 \\
\hline SumH & Grupo 1 & 26 & 0,00 & 5,00 & 2,00 & 1,36 & 2,00 & 2,00 & $\mathrm{U}=283,00$ & 0,95 \\
\hline Sйा11 & Grupo 2 & 22 & 0,00 & 6,00 & 2,09 & 1,57 & 2,00 & 1,00 & $\mathrm{z}=-0,07$ & D, \\
\hline Isolamento & Grupo 1 & 26 & 0,00 & 0,64 & 0,14 & 0,16 & 0,14 & 0,00 & $\mathrm{U}=246,00$ & 0,40 \\
\hline ssoramento & Grupo 2 & 22 & 0,00 & 1,00 & 0,23 & 0,28 & 0,13 & 0,00 & $\mathrm{z}=-0,85$ & 0,40 \\
\hline Food & Grupo 1 & 26 & 0,00 & 1,00 & 0,04 & 1,20 & 0,00 & 0,00 & $\mathrm{U}=258,00$ & 0,23 \\
\hline Food & Grupo 2 & 22 & 0,00 & 1,00 & 0,14 & 0,35 & 0,00 & 0,00 & $\mathrm{z}=-1,21$ & 0,23 \\
\hline $\mathrm{COP}$ & Grupo 1 & 26 & 0,00 & 1,00 & 0,23 & 0,43 & 0,00 & 0,00 & $\mathrm{U}=259,00$ & 0,41 \\
\hline COP & Grupo 2 & 22 & 0,00 & 1,00 & 0,14 & 0,35 & 0,00 & 0,00 & $\mathrm{z}=-0,83$ & 0,41 \\
\hline$A G$ & Grupo 1 & 26 & 0,00 & 3,00 & 0,38 & 0,70 & 0,00 & 0,00 & $\mathrm{U}=277,00$ & 0.81 \\
\hline & Grupo 2 & 22 & 0,00 & 3,00 & 0,36 & 0,73 & 0,00 & 0,00 & $\mathrm{z}=-0,24$ & 0,01 \\
\hline GHR & Grupo 1 & 26 & 0,00 & 1,00 & 0,04 & 0,20 & 0,00 & 0,00 & $\mathrm{U}=275,00$ & 036 \\
\hline UHк & Grupo 2 & 22 & 0,00 & 0,00 & 0,00 & 0,00 & 0,00 & 0,00 & $\mathrm{z}=-0,92$ & 0,50 \\
\hline PHR & Grupo 1 & 26 & 0,00 & 1,00 & 0,85 & 0,37 & 0,00 & 1,00 & $\mathrm{U}=252,00$ & 0,32 \\
\hline (110 & Grupo 2 & 22 & 0,00 & 0,46 & 0,73 & 0,46 & 0,00 & 1,00 & $z=-1,00$ & L \\
\hline PER & Grupo 1 & 26 & 0,00 & 1,00 & 0,19 & 0,40 & 0,00 & 0,00 & $\mathrm{U}=283,00$ & 0,93 \\
\hline $1 \mathrm{LN}$ & Grupo 2 & 22 & 0,00 & 1,00 & 0,18 & 0,39 & 0,00 & 0,00 & $\mathrm{z}=-0,09$ & ני, \\
\hline
\end{tabular}




\section{Discussão}

Como já mencionado, o processo de avaliação psicológica de crianças contribui para identificar as fraquezas e as suas potencialidades e sugerir os encaminhamentos adequados (Schelini, Gomes, \& Wechsler, 2006). O processo de avaliação de crianças quando realizado precocemente favorece o seu desenvolvimento, tendo um caráter preventivo (Perez-Ramos, 2000). Neste sentido, cabe destacar que a avaliação do relacionamento interpessoal de crianças pode favorecer a compreensão de como essas estabelecem seus vínculos, se são próximos ou distantes, se as crianças têm capacidade de empatia, como estabelecem as relações e identificações com os demais. Esses fatores auxiliam na formação de uma identidade estável, e a maneira como se manifestam contribui para a identificação de comportamentos psicopatológicos (Weiner, 2000).

No Brasil o número de testes que podem ser utilizados com a população de crianças não é tão extenso, sendo necessário investir em pesquisas com instrumentos diversos que apresentem estímulos diferentes, o que permite vários modos de expressão das características avaliadas e, portanto a apreensão mais completa e profunda do problema. Por essa razão o objetivo deste estudo foi verificar se os resultados dos indicadores de relacionamento interpessoal no Teste ZSC diferem as crianças identificadas como mais populares ou menos populares pelo sociograma.

Os resultados confirmaram algumas das hipóteses geradoras deste trabalho. $\mathrm{O}$ grupo de crianças populares apresentou aumento estatisticamente significativo de $p$ e Mp. O indicador M (movimento humano), conforme já mencionado, é descrito por Weiner (2000) como a capacidade de empatia, a habilidade de se colocar no lugar dos demais, pressupondo a ocorrência de impressões mais realísticas a respeito dos outros e das relações estabelecidas. Destaca-se que o indicador movimento humano (M) também se apresentou aumentado em outras pesquisas como na de Lis, Magro e Rossi (1990), que constataram que a medida que, as crianças amadurecem, aumenta a média de M. Cita-se como comparação o estudo de Uhinki, Mattlar, Sandahl, Vesala e Carlsson (1990), que verificaram que a média de $\mathrm{M}$ foi um pouco menor no grupo de adolescentes $(\mathrm{X}=1,3)$ quando comparado ao grupo de adultos $(X=1,5)$, o que é esperado, pois estes ainda não completaram o desenvolvimento afetivo e emocional. Ainda com relação ao M, Tavella e Villemor-Amaral (2014) identificaram seu aumento em crianças mais criativas. Na mesma direção a pesquisa de Resende, Carvalho e Martins (2012), embora com o Método de
Rorschach, constataram que os adolescentes de 12 a 14 anos também apresentaram aumento de respostas $\mathrm{M}$, quando comparados aos outros dois grupos de crianças de 5-7 e 8-11 anos, indicando que com o passar dos anos a maneira de se relacionar e perceber os demais tende a melhorar. Todos esses resultados indicam uma tendência de que as crianças ao longo do desenvolvimento ampliam a capacidade de empatia, colocando-se no lugar dos outros, com representações das relações mais de acordo com a realidade. Pressupõese, portanto, que esta característica revele maior maturidade nas relações interpessoais, pois o individuo está mais voltado para o coletivo e não somente para as suas necessidades individuais. Isso corrobora uma das hipóteses do presente estudo, de que as crianças populares, quando comparadas às menos populares tendem a se relacionar com os demais e demonstram interesse nas relações estabelecidas de forma mais enfática que as crianças menos populares, evidenciadas pelo aumento de $\mathrm{M}$.

$\mathrm{O}$ indicador $\mathrm{M}$ relaciona-se também, do ponto de vista da interpretação com habilidades cognitivas que facilitam conceber soluções para os problemas (Villemor-Amaral \& Primi, 2009; Weiner, 2000). Portanto, mais uma vez, confirma-se uma habilidade importante para um convívio social mais harmônico. Entretanto, esperava-se também aumento de Movimentos ativos nas crianças populares, por ser este um melhor representante de autonomia, no caso de adultos. O que se observou, porém, foi que as crianças populares quando comparadas às crianças menos populares, revelaram uma média significativamente maior de $\operatorname{Mp}(\mathrm{X}=0,35, p=0,04)$ e de $\mathrm{p}$ em geral $(\mathrm{X}=0,62, p=0,05)$. Isso poderia sugerir tendência maior passividade nessas crianças, porém tal não se confirma quando o que se observa é que a frequência absoluta de movimentos humanos ativos é maior que a de passivos, embora não com nível de significância adequado. Esse resultado assemelha-se ao obtido na pesquisa de Sandahl, Mattlar, Carlson, Vesala e Rosenqvist (1990), quando identificaram que os adultos apresentaram maior proporção do ativo comparado ao passivo (17:9). Nesta perspectiva, compreende-se que o Mp elevado deve-se primordialmente ao fato de as crianças mais populares terem maior quantidade de respostas de $\mathrm{M}$ em geral, independentemente de serem passivos ou ativos.

Outro indicador cujo resultado corrobora as hipóteses foi conteúdo humano inteiro $(\mathrm{PureH}) \mathrm{com}$ média maior, marginalmente significativa, no grupo de crianças populares $(\mathrm{X}=1,35, p=0,06)$. De acordo com Villemor-Amaral e Primi (2009), PureH revela identificação mais clara e estável com as pessoas, maior 
interesse pelos outros e pelas relações interpessoais. Lis, Magro e Rossi (1990) também demonstraram que com o passar da idade há um aumento das médias de PureH. Em outro estudo, Grazziotin e Scortegagna (2012) aplicaram e correlacionaram o ZSC e o IHS na população adulta, encontrando o aumento das habilidades sociais gerais, acompanhando aumento de PureH. Resende, Carvalho e Martins (2012) aplicaram o Método de Rorschach e constataram que os adolescentes de 12 a 14 anos apresentaram aumento dessa mesma variável, quando comparados aos outros grupos de 5-7 anos e 8-11 anos. Deste modo, confirmase que a PureH revela interesse pelas pessoas e uma visão mais integrada e realista, podendo favorecer as relações interpessoais, justificando maior incidência desse indicador entre as crianças mais populares.

Outra análise realizada refere-se à proporcionalidade dos diversos tipos de conteúdos humanos no teste. As crianças menos populares apresentaram resultado estatisticamente significativo em $\mathrm{H}<\mathrm{Hd}+(\mathrm{H})+(\mathrm{Hd})$, revelando redução das figuras humanas inteiras, em relação ao conteúdo para-humano, detalhe humano e detalhe para-humano. Nessa direção, a pesquisa de Balbi e Queiroz (2012) com o Método de Rorschach constatou que os adultos que apresentaram valores positivos no CDI, que inclui a proporção mencionada acima, tinham dificuldades de relacionamento, evidenciadas também nas respostas dadas nas entrevistas. Villemor-Amaral e Primi (2009) destacam que o aumento dessa proporção sugere uma percepção fantasiosa dos demais, certa limitação na percepção dos outros por inteiro, favorecendo a inabilidade social. Isso corrobora o esperado teoricamente para esse indicador, auxiliando compreender as dificuldades das crianças menos populares.

\section{Conclusão}

Esta pesquisa atingiu o objetivo pretendido, comprovar evidências de validade para o ZSC, na avaliação de relacionamento interpessoal de criança, averiguando uma possível associação entre os resultados de um sociograma e o desempenho das crianças nos indicadores de relacionamento interpessoal do ZSC. Os resultados demonstram que quatro indicadores do ZSC diferenciam crianças mais populares e menos populares. Esses indicadores estão claramente associados na literatura com melhor qualidade nos relacionamentos interpessoais, sendo frequentemente considerados sinal de maior amadurecimento.

Tendo em vista as dificuldades de se encontrar evidências de validade para as principais interpretações nas técnicas expressivas, essa pesquisa trouxe uma contribuição importante nessa direção. Verificou-se que o teste de ZSC foi capaz de discriminar crianças populares e menos populares por meio dos indicadores previstos nas hipóteses norteadoreas deste trabalho. Sendo assim, as crianças populares apresentaram maior capacidade de empatia, apesar da imaturidade típica da idade e identificação com figuras humanas, e as crianças menos populares demonstraram mais dificuldades em se identificarem com figuras humanas reais e inteiras. Conclui-se, portanto, que o ZSC, em certa medida, mostrou-se útil para identificar habilidade de relacionamento interpessoal em crianças, mas sobretudo confirmou-se mais uma vez a validade das interpretações atribuídas aos indicadores de relacionamento interpessoal nesse teste.

Cabe, finalmente, mencionar as limitações deste estudo, decorrentes da dificuldade de se obter uma amostra maior em razão da própria natureza da pesquisa que envolve técnica expressiva da personalidade. Por um lado, o modo de aplicação individual desse teste aumenta significativamente o tempo de coleta de dados e quanto maior o tempo dispendido maior a perda de voluntários ao longo do caminho. Por outro lado, a pesquisa com menores de idade, tanto depende do consentimento das escolas, quanto dos pais e da própria criança. Portanto, embora, o objetivo tenha sido atingido, certamente novos estudos com amostras mais amplas contribuirão para maior desenvolvimento desse teste.

\section{Referências}

American Educational Rersearch Association, American Psychological Association National Council On Measurement In Education. (1999). Standards for Educational and Psychological Testing. Washington, DC: AERA.

Anzieu, D. (1989). Os métodos projetivos (5a ed.). Rio de Janeiro: Campus.

Balbi Neto, R. R. Q. \& Queiroz, S. S. (2012). Indícios de validade do déficit relacional no Método de Rorschach para população adulta não paciente. Psico-USF, 17(3), 475-484. http://dx.doi.org/10.1590/S1413-82712012000300014

Bandeira, D. R, Trentini, C. M., Winck, G. E. \& Lieberknecht, L. (2006) Considerações sobre as técnicas projetivas no contexto atual. In A. P. Noronha, A. A. A. Santos, \& F. F. Sisto (Org.). Facetas do Fazer em Avaliação Psicológica (pp. 125-138). São Paulo: Vetor. 
Bartholomeu, D., Carvalho, L. F., Silva, M. C. R., Miguel, F. K., \& Machado, A. A. (2011). Aceitação e rejeição entre pares e habilidades sociais em universitários. Estudos de Psicologia, 16(2), 155-162. http://dx.doi.org/10.1590/S1413294X2011000200006

Bartholomeu, D., Montiel, J. M., \& Pessotto, F. (2011). Sociometria e habilidades sociais em alunos do ensino médio. Estudos Interdisciplinares em Psicologia, 2(2), 211-228. http://dx.doi.org/10.5433/2236-6407.2011v2n2p211

Davoglio, T. R., Gauer, G. J. C., Jaeger, J. V. H., \& Tolotti, M. D. (2012). Personalidade e psicopatia: implicações diagnósticas na infância e adolescência. Estudos de Psicologia, 17(3), 453-460. http://dx.doi.org/10.1590/S1413294X2012000300014

Exner, J. E. \& Sendin, C. (1999). Manual de interpretação do Rorschach para o sistema compreensivo. São Paulo: Casa do Psicólogo.

Grazziotin, J. B. D. \& Scortegagna, S. A. (2012). Zulliger e habilidade social: evidências de validade no contexto empresarial. Psicologia: Reflexão e Crítica, 25(1), 69-78. http://dx.doi.org/10.1590/s0102-79722012000100009

Hisatugo, C. L. \& Custódio, E. M. (2013). Avaliação psicológica e da personalidade e o estudo normativo do Rorschach para o uso em crianças brasileiras. Psicólogo inFormação, 17, 79-86. http://dx.doi.org/10.15603/2176-0969/ pi.v17n17p79-86

Landis, J. R. \& Koch, G. G. (1977). The measurement of observer agreement for categorical data. Biometrics, 33(1), 159-174. http://dx.doi.org/10.2307/2529310

Lis, A., Magro, T. \& Rossi, G. (1990). A comparison between the Zulliger Test individually administered and the Rorschach Test in children aged 6-11. British Journal of Projective Psychology, 35(2), 35-48.

Moreno, J. L. (2008). Quem sobreviverá? Fundamentos da sociometria, da psicoterapia e do sociodrama [Who shall survive? Foundations of sociometry group psychotherapy and sociodrama] (M. Aguiar, Trad.). São Paulo: Daimon (Trabalho original publicado em 1953).

Nunes, M. L. T, Teixeira, R. P., \& Deakin, E. K. (2010). Técnicas projetivas e avaliação de psicoterapia psicanalítica com crianças. In M. L. T. Nunes (Org.). Técnicas projetivas com crianças (pp. 145-169). São Paulo: Casa do Psicólogo.

Perez-Ramos, A. L. Q (2000). Avaliação prospectiva: o exame precoce da criança. In J. A. Cunha. Psicodiagnóstico V (pp. 151-157). Porto Alegre: Artmed.

Primi, R., Muniz, M., \& Nunes, C. H. S. S. (2009). Definições contemporâneas de validade de testes psicológicos. In C. S. Hutz (Org.). Avanços polêmicos em avaliação psicológica (pp. 243-265). São Paulo: Casa do Psicólogo.

Resende, A. C., Carvalho, T. C. R., \& Martins, W. (2012). Desempenho Médio de Crianças e Adolescentes no Método de Rorschach Sistema Compreensivo. Avaliação Psicológica, 11(3), 375-394.

Sandahl, C., Mattlar, C. E, Carlsson, A., Vesala, P., \& Rosenqvist, A. (1990) The personality structure for the normal adults, as revealed by the Zulliger. British Journal of Projective Psychology, 35(2), 54-60.

Schelini, P. W., Gomes, V. L. T., \& Wechsler, S. M. (2006). Avaliação psicológica infantil: aspectos cognitivos e neuropsicológicos. In A. P. Noronha, A. A. A. Santos, \& F. F. Sisto (Org.). Facetas do Fazer em Avaliação Psicológica (pp. 81-94). São Paulo: Vetor.

Tavares, M. (2003). Validade clínica. Psico-USF, 8(2), 125-136.

Tavella, R. R. \& Villemor-Amaral, A. E. (2014). O teste de Zulliger-SC: avaliação da criatividade em crianças. Estudos de Psicologia, 31(4) , 489-497. http://dx.doi.org/10.1590/0103-166X2014000400003

Uhinki, A., Mattlar, C. E., Sandahl, P., Vesala, A., \& Carlsson (1990). Personality traits characteristic for adolescents highlighted by the Zulliger. British Journal of Projective Psychology, 35(2), 49-53.

Urbina, S. (2007). Fundamentos da testagem psicológica. Porto Alegre: Artmed.

Vaz, C. E. (1998). Z-teste: técnica de Zulliger - forma coletiva. São Paulo: Casa do Psicólogo.

Viglione, D. J., Perry, W., \& Meyer, G. J. (2003). Refinements in the Rorschach Ego Impairment Index incorporating the Human Representational Variable. Journal of Personality Assessment, 81, 149-156. http://dx.doi.org/10.1207/ S15327752JPA8102 06

Villemor-Amaral, A. E. \& Primi, R. (2009). O Teste de Zulliger no Sistema Compreensivo (ZSC): forma individual. São Paulo: Casa do Psicólogo.

Weiner, I. B. (2000). Princípios da interpretação do Rorschach. São Paulo: Casa do Psicólogo.

Zulliger, H. \& Salomon, F. (1970). El test de Zulliger: un test individual y colectivo. Buenos Aires: Kapelusz.

\author{
Autores: \\ Fabiola Cristina Biasi - Mestre, Universidade São Francisco. \\ Anna Elisa de Villemor-Amaral - Doutora, Universidade São Francisco. \\ Endereço para correspondência: \\ Fabiola Cristina Biasi \\ <fa_biasi@yahoo.com.br> \\ Recebido em: 03.10.2015 \\ Aceito em: 03.12.2015
}

